\title{
Postprocedural, midterm, and long-term results of cerebral aneurysms treated with flow-diverter devices: 7-year experience at a single center
}

\author{
Francesco Briganti, MD, ${ }^{1}$ Giuseppe Leone, MD, ${ }^{2}$ Luigi Cirillo, MD, ${ }^{3}$ Oreste de Divitiis, MD, ${ }^{4}$ \\ Domenico Solari, MD, PhD, ${ }^{4}$ and Paolo Cappabianca, $\mathrm{MD}^{4}$
}

\begin{abstract}
1 Unit of Interventional Neuroradiology, Department of Advanced Biomedical Sciences; ${ }^{2}$ Department of Advanced Biomedical Sciences; ${ }^{4}$ Division of Neurosurgery, Department of Neurosciences, Reproductive and Odontostomatological Sciences, Università degli Studi di Napoli Federico II, Napoli; and ${ }^{3}$ Neuroradiology Department, IRCCS Institute of Neurological Sciences of Bologna, Bellaria Hospital, Bologna, Italy
\end{abstract}

OBJECTIVE Flow diversion has emerged as a viable treatment option for selected intracranial aneurysms and recently has been gaining traction. The aim of this study was to evaluate the safety and effectiveness of flow-diverter devices (FDDs) over a long-term follow-up period.

METHODS The authors retrospectively reviewed all cerebral aneurysm cases that had been admitted to the Division of Neurosurgery of the Università degli Studi di Napoli between November 2008 and November 2015 and treated with an FDD. The records of 60 patients (48 females and 12 males) harboring 69 cerebral aneurysms were analyzed. The study end points were angiographic evidence of complete aneurysm occlusion, recanalization rate, occlusion of the parent artery, and clinical and radiological evidence of brain ischemia. The occlusion rate was evaluated according to the O'Kelly-Marotta (OKM) Scale for flow diversion, based on the degree of filling (A, total filling; B, subtotal filling; C, entry remnant; D, no filling). Postprocedural, midterm, and long-term results were strictly analyzed.

RESULTS Complete occlusion (OKM D) was achieved in 63 (91\%) of 69 aneurysms, partial occlusion (OKM C) in 4 $(6 \%)$, occlusion of the parent artery in $2(3 \%)$. Intraprocedural technical complications occurred in 3 patients $(5 \%)$. Postprocedural complications occurred in 6 patients (10\%), without neurological deficits. At the 12-month follow-up, 3 patients $(5 \%)$ experienced asymptomatic cerebral infarction. No further complications were observed at later follow-up evaluations (> 24 months). There were no reports of any delayed aneurysm rupture, subarachnoid or intraparenchymal hemorrhage, ischemic complications, or procedure- or device-related deaths.

CONCLUSIONS Endovascular treatment with an FDD is a safe treatment for unruptured cerebral aneurysms, resulting in a high rate of occlusion. In the present study, the authors observed effective and stable aneurysm occlusion, even at the long-term follow-up. Data in this study also suggest that ischemic complications can occur at a later stage, particularly at 12-18 months. On the other hand, no other ischemic or hemorrhagic complications occurred beyond 24 months. https://thejns.org/doi/abs/10.3171/2017.3.FOCUS1732

KEY WORDS intracranial aneurysm; flow-diverter devices; Pipeline embolization device; p64 flow-modulation device; flow-redirection endoluminal device; endovascular treatment

$\mathrm{F}$ LOW-DIVERTER DEVICES (FDDs) are new-generation stents placed in the parent artery at the level of the aneurysm neck. Indeed, they disrupt intraaneurysmal flow providing significant rheological effects with potential changes in the transmural pressure gradient. In a second stage, progressive intraaneurysmal thrombosis starts offering good support for the development of a neointima. ${ }^{15}$
Thus, flow diversion may provide a valid alternative to conventional endovascular techniques, especially when dealing with difficult-to-treat aneurysms such as widenecked, fusiform, or giant aneurysms or those with complex morphology, cases in which an FDD provides the possibility of preserving the parent artery. 1,2,3-10,13,17-19,22-25 Although the introduction of these devices is quite recent, experience with them is rapidly increasing. However, what

ABBREVIATIONS ACA = anterior cerebral artery; CTA = CT angiography; DSA = digital subtraction angiography; FDD = flow-diverter device; ICA = internal carotid artery; $\mathrm{MCA}=$ middle cerebral artery; MRA = MR angiography; $m R S$ = modified Rankin Scale; OKM = O'Kelly-Marotta; PAO = parent artery occlusion; PCoA = posterior communicating artery; PED = Pipeline embolization device; PICA = posterior inferior cerebellar artery; SAH = subarachnoid hemorrhage; SCA = superior cerebellar artery.

SUBMITTED January 25, 2017. ACCEPTED March 28, 2017.

INCLUDE WHEN CITING DOI: 10.3171/2017.3.FOCUS1732. 
constitutes the best endovascular procedure and the indications for using an FDD are still matters of debate.

Since the approval of the Pipeline embolization device (PED) and the SILK flow diverter, new FDDs have been introduced in Europe. These new devices such as Pipeline Flex, FRED (flow redirection endoluminal device), and p64 flow modulation device meet the requirements for modern second-generation devices. In particular, they have been structured to avoid device malpositioning and to reduce the rate of device-related complications. Their main characteristics include an improved delivery system with easier and more precise deployment, mesh porosity for maintaining flow in branching vessels, and good radiopacity for confirming apposition to the vessel wall and avoiding unexpected thrombus formation. Therefore, the safety and efficacy profiles associated with each of these FDDs, even on long-term follow-up, must be understood.

\section{Methods \\ Study Design}

This study was approved by the institutional review board of the School of Medicine of Università degli Studi di Napoli Federico II, which waived the necessity for informed consent given the retrospective nature of the study. Written informed consent was obtained from the patients prior to any invasive clinico-diagnostic or surgical procedure; indeed, it was obtained for the eventual anonymous publication of any patient records and/or information for scientific purposes.

We retrospectively reviewed all cerebral aneurysms cases that had been admitted to the Division of Neurosurgery of the Università degli Studi di Napoli Federico II between November 2008 and November 2015 and treated with an FDD. Study inclusion criteria consisted of no previous neurosurgical treatment, aneurysms difficult to treat with other techniques (neurosurgical and endovascular) because of their anatomical configuration, and patients with complete clinical and radiological pre- and postprocedural assessment. All patients underwent CT angiography (CTA) using native images, 2D multiplanar reconstructions, and 3D volume rendering; digital subtraction angiography (DSA) was performed in all patients as well. The aneurysm location and size, aneurysm neck, and neck/sac ratio were noted for each patient. Aneurysm size was classified as small $(<15 \mathrm{~mm})$, large $(16-25 \mathrm{~mm})$, and giant (>25 mm).

The study end points were angiographic evidence of complete aneurysm occlusion, recanalization rate, occlusion of the parent artery, and clinical and radiological evidence of brain ischemia. The occlusion rate was evaluated according to the O'Kelly-Marotta (OKM) Scale for flow diversion, based on the degree of filling (A, total filling; $\mathrm{B}$, subtotal filling; C, entry remnant; D, no filling). ${ }^{20} \mathrm{We}$ strictly analyzed postprocedural, midterm, and long-term results.

\section{Flow-Diverter Devices: Technical Considerations}

Six types of FDDs are currently approved for the treatment of intracranial aneurysms in clinical practice: PEP (Covidien), SILK flow diverter (Balt Extrusion), FRED (Microvention), the p64 flow modulation device (Phenox),
Surpass Streamline flow diverter (Stryker), and Derivo embolization device (Acandis).

The PED is a flexible, braided mesh tube of 48 interwoven microfilaments consisting of $25 \%$ platinum-tungsten and $75 \%$ cobalt-chromium-nickel alloy, designed to provide $30 \%-35 \%$ metal coverage of the inner surface of the target vessel. When fully deployed, the device remains very flexible and can adapt to tortuous vessel anatomy. The PED received approval for clinical use in Europe in 2008 and in the United States in 2011. The second-generation PED, named Pipeline Flex, received the European CE mark of approval in March 2014 and subsequently received FDA approval in February 2015. Its design includes numerous delivery system changes to enhance device opening and provide additional safety with a re-sheathing feature $(70 \%-80 \%$ retrievability).

The SILK flow diverter is also a flexible mesh stent constructed from 48 braided nitinol and 4 platinum microfilaments, with a pore size of $110-250 \mathrm{~mm}$ and $35 \%-55 \%$ metal coverage at its nominal vessel diameter. This device was approved for clinical use in Europe in 2008.

The FRED system is a self-expanding nickel titanium paired stent, designed with integrated dual-layer coverage with a low-porosity inner mesh of higher pore attenuation (48 nitinol wires) and an outer stent with high porosity (16 nitinol wires); its structure facilitates simultaneous deployment and partial re-sheathability by a single operator (70\%-80\% retrievability).

The p64 flow modulation device is a flexible, meshlike tube of 64 interwoven microfilaments completely recoverable with 8 proximal radiopaque bundle markers. Because of its controlled mechanical detachment, the p64 can be retrieved into the microcatheter and removed or repositioned until $100 \%$ of its length has been extruded.

The Surpass Streamline flow diverter is made of a cobalt-chromium alloy, with multiple fine fibers that are braided into a flexible mesh tube and a variable number of braiding wires according to the device size.

The Derivo is a new FDD consisting of 48 braiding nitinol wires with a radiopaque platinum core.

All of the devices are delivered via a microcatheter (0.027-inch for Pipeline, SILK, FRED, Derivo, and Surpass; 0.023-inch for SILK) and require a 6- or 7-Fr guide catheter. The usual insertion technique, common to the all FDDs, involves deploying the distal tip of a delivery microcatheter beyond the aneurysm neck and then pushing the FDD to the tip of the delivery wire. The system is then aligned with the aneurysm under fluoroscopy so that the FDD is deployed and the microcatheter is withdrawn. This requires a perfect combination of pushing the delivery wire and retrieving the microcatheter to allow the FDD to expand correctly and eventually compensate for any foreshortening.

\section{Patient Characteristics}

Sixty patients (48 females [80\%] and 12 males [20\%]) harboring 69 cerebral aneurysms who had undergone endovascular treatment with FDDs were eligible for inclusion in this study. The mean patient age was 57 years (range 33-76 years). Fifty-four patients (90\%) were diagnosed with unruptured aneurysms, and $6(10 \%)$ presented 
with ruptured aneurysms. In the latter 6 patients, the hemorrhagic episode occurred between 15 and 30 days before FDD treatment. Three patients had Hunt and Hess Grade I subarachnoid hemorrhage (SAH) and 3 had Grade IA SAH (with third and sixth cranial nerve palsy, respectively). Among the 54 patients with no previous hemorrhage, $16(27 \%)$ complained of headache, $11(18 \%)$ had cranial nerve palsies, and 2 (3\%) presented with hemiparesis due to direct aneurysm compression (Table 1).

\section{Aneurysm Characteristics}

Of the 69 aneurysms, 64 (93\%) were in the anterior circulation and $5(7 \%)$ in the posterior circulation. The most common localization was the paraophthalmic internal carotid artery (ICA; 36 [52\%]), followed by the posterior communicating artery (PCoA; 14 [20\%]) and intracavernous ICA (7 [10\%]; Table 1); 6 aneurysms (9\%) were located at the $\mathrm{M}_{1}-\mathrm{M}_{2}$ segments of the middle cerebral artery (MCA) and 1 aneurysm (2\%) was located at the $\mathrm{A}_{1}-\mathrm{A}_{2}$ segments of the anterior cerebral artery (ACA). Among the posterior circulation aneurysms, $2(3 \%)$ were located at the superior cerebellar artery (SCA), 2 (3\%) were at the basilar artery, and $1(1 \%)$ was at the posterior inferior cerebellar artery (PICA).

Sixty-seven aneurysms were saccular and 2 were fusiform. Aneurysm size ranged from 3 to $27 \mathrm{~mm}$ (median diameter $10 \mathrm{~mm}$ ). Fifty-four aneurysms (78\%) were small, $13(19 \%)$ were large, and $2(3 \%)$ were giant. The aneurysm neck size ranged from 2 to $12 \mathrm{~mm}$ (average $6 \mathrm{~mm}$ ). The neck/sac ratio ranged from 0.5 to 1 (average 0.8 ; Table 1 ).

\section{Endovascular Treatment and Medication}

The FDD was the unique first-line treatment for 64 aneurysms (93\%). In 2 cases (3\%), the device was implanted in association with coils because of complex aneurysm morphology and size. Another 2 aneurysms (3\%), previously treated with endovascular coiling, were subsequently retreated with an FDD because of recanalization. In 1 case (1\%), the FDD was deployed after other devices (LEO stent, Balt Extrusion). Among the 63 implanted FDDs used to treat 69 aneurysms, 31 were PED, 4 SILK, 23 FRED, and 5 p64.

Once the appropriate treatment was decided, each patient preoperatively received $75 \mathrm{mg}$ of clopidogrel daily for 5 days in association with $150 \mathrm{mg}$ of aspirin. Patients treated in the subacute phase of SAH received a loading dose of clopidogrel $(600 \mathrm{mg}) 4$ hours before the procedure. The thrombocyte inhibition test was not routinely performed. All patients had continuous intravenous infusion of heparin during the procedure (70 IU/kg as a bolus) and a rush of 1000 IU every hour during the procedure, to maintain an activated clotting time (ACT) $>250-300$ seconds. The clopidogrel $(75 \mathrm{mg})$ and aspirin $(100 \mathrm{mg})$ were administered daily for up to 6 months after the procedure for anterior circulation aneurysms and up to 12 months for posterior circulation aneurysms; thereafter, only the aspirin was continued. The senior interventional neuroradiologist (F.B.) performed all endovascular treatments. The procedure was performed under general anesthesia through catheterization of the right common femoral ar-
TABLE 1. Clinicoradiological characteristics in 60 patients with 69 aneurysms

\begin{tabular}{|c|c|}
\hline Characteristic & No. \\
\hline \multicolumn{2}{|l|}{ Sex } \\
\hline $\mathrm{F}$ & $48(80 \%)$ \\
\hline M & $12(20 \%)$ \\
\hline Average age in yrs (range) & $57(33-76)$ \\
\hline \multicolumn{2}{|l|}{ Clinical presentation } \\
\hline $\mathrm{SAH}$ & $6(10 \%)$ \\
\hline No SAH & $54(90 \%)$ \\
\hline Headache & $16(27 \%)$ \\
\hline Cranial nerve palsies & $11(18 \%)$ \\
\hline Hemiparesis & $2(3 \%)$ \\
\hline Other unrelated symptoms & $25(42 \%)$ \\
\hline \multicolumn{2}{|l|}{ Aneurysm location } \\
\hline Paraophthalmic ICA & $36(52 \%)$ \\
\hline PCoA & $14(20 \%)$ \\
\hline Cavernous ICA & $7(10 \%)$ \\
\hline$A_{1} / A_{2}$ & $1(1 \%)$ \\
\hline MCA & $6(9 \%)$ \\
\hline Basilar artery & $2(3 \%)$ \\
\hline PICA & $1(1 \%)$ \\
\hline SCA & $2(3 \%)$ \\
\hline \multicolumn{2}{|l|}{ Aneurysm size } \\
\hline Small (<15 mm) & $54(78 \%)$ \\
\hline Large (16-25 mm) & $13(19 \%)$ \\
\hline Giant (>25 mm) & $2(3 \%)$ \\
\hline \multicolumn{2}{|l|}{ Aneurysm neck/sac ratio } \\
\hline 0.5 & $14(20 \%)$ \\
\hline 0.6 & $13(19 \%)$ \\
\hline 0.7 & $16(23 \%)$ \\
\hline 0.8 & $7(10 \%)$ \\
\hline 0.9 & $11(16 \%)$ \\
\hline 1.0 & $6(9 \%)$ \\
\hline Fusiform & $2(3 \%)$ \\
\hline
\end{tabular}

tery by using an 8 -Fr vascular sheath and a triaxial system. The FDD size was chosen according to the proximal parent vessel diameter. The device was then placed at the level of the aneurysm. Correct apposition to the vessel wall was assessed with DSA and nonsubtracted angiographic images. The procedure was considered successful if the FDD completely covered the aneurysm. Intraaneurysmal contrast stasis was observed in all patients immediately after the procedure.

\section{Follow-Up}

Clinical and neurological evaluations were performed immediately after the procedure, at discharge, and at 1 , $3,6,12$, and 24 months after the procedure. Radiological examination with MR angiography (MRA) or CT was performed at 3-6 and 12 months after the procedure and thereafter until evidence of aneurysm occlusion; in all cases, complete aneurysm occlusion was then confirmed on DSA. For patients with documented occlusion, cerebral contrast-enhanced MRA was performed every 12 months.

The actual follow-up ranged between 3 and 84 months (median 50 months). All patients underwent at least a 12 -month follow-up. Fifty of them (83\%) were also studied 
TABLE 2. Data on aneurysm occlusion among 69 aneurysms

\begin{tabular}{|c|c|c|c|c|}
\hline \multirow[b]{2}{*}{ Parameter } & \multirow[b]{2}{*}{ No. } & \multicolumn{3}{|c|}{ No. } \\
\hline & & Complete Occlusion & Partial Occlusion & Parent Vessel Occlusion \\
\hline \multicolumn{5}{|c|}{ Occlusion rate (69 aneurysms) } \\
\hline Complete (OKM D) & $63(91 \%)$ & & & \\
\hline Partial (OKM C) & $4(6 \%)$ & & & \\
\hline Parent vessel occlusion & $2(3 \%)$ & & & \\
\hline \multicolumn{5}{|c|}{ Time of complete occlusion (63 aneurysms) } \\
\hline $3 \mathrm{mos}$ & $34(49 \%)$ & & & \\
\hline $6 \mathrm{mos}$ & $20(29 \%)$ & & & \\
\hline $12 \mathrm{mos}$ & $8(12 \%)$ & & & \\
\hline $18 \mathrm{mos}$ & $1(1 \%)$ & & & \\
\hline \multicolumn{5}{|c|}{ Occlusion related to aneurysm location } \\
\hline Paraophthalmic ICA & 36 & 35 & 0 & 1 \\
\hline Cavernous ICA & 7 & 7 & 0 & 0 \\
\hline $\mathrm{PCOA}$ & 14 & 13 & 1 & 0 \\
\hline$A_{1} / A_{2}$ & 1 & 0 & 1 & 0 \\
\hline MCA & 6 & 5 & 1 & 0 \\
\hline Basilar artery & 2 & 1 & 0 & 1 \\
\hline PICA & 1 & 1 & 0 & 0 \\
\hline SCA & 2 & 1 & 1 & 0 \\
\hline
\end{tabular}

at 24 and 36 months, $44(73 \%)$ at 48 months, $35(58 \%)$ at 60 months, $30(50 \%)$ at 72 months, $25(42 \%)$ at 84 months.

\section{Results}

Complete occlusion (OKM D) was achieved in 63 aneurysms (91\%): at 3 months in 34 cases (49\%), at 6 months in 20 cases (29\%), at 12 months in 8 cases (12\%), and at 18 months in 1 case (1\%; Table 2). Partial occlusion (OKM C) occurred in 4 aneurysms (6\%), and parent artery occlusion (PAO) occurred in $2(3 \%)$. Among the 4 cases with partial occlusion, 1 was an aneurysm of the $A_{1}-A_{2}$ segments, 1 was located at the MCA, 1 was at the PCoA, and 1 was at the SCA. A PAO occurred in the case of a large basilar artery fenestration aneurysm at the 3-month follow-up because of dual antiplatelet therapy withdrawal. The second case of PAO was iatrogenic, occurring after a severe vasospasm. Overall, the clinical conditions were stable so that all patients were classified as having a modified Rankin Scale (mRS) score of 0, except for 1 patient, who presented with an mRS score of 3 at both the pre- and postoperative assessments.

TABLE 3. Correlation of occlusion time with aneurysm size among 69 aneurysms

\begin{tabular}{lcrcc}
\hline & No. of & \multicolumn{4}{c}{ Aneurysm Size } \\
\cline { 3 - 5 } Occlusion \& Timing & Aneurysms & Small & Large & Giant \\
\hline Complete at 3 mos & 34 & 32 & 2 & \\
\hline Complete at 6 mos & 21 & 16 & 5 & \\
\hline Complete at 12 mos & 7 & 1 & 5 & 1 \\
\hline Complete at 18 mos & 1 & & & 1 \\
\hline Partial (OKM C) & 4 & 4 & & \\
\hline Parent vessel occlusion & 2 & 1 & 1 & \\
\hline Total & 69 & 54 & 13 & 2 \\
\hline
\end{tabular}

Sixty-one (97\%) of the 63 implanted FDDs were patent at the last follow-up.

An analysis of the correlation between occlusion time and aneurysm size (Table 3) showed a high rate of occlusion among small aneurysms at 3 months after the procedure (46\%) and no significant difference in occlusion time according to aneurysm size.

Intraprocedural technical complications occurred in 3 patients (5\%): detachment failure in 2 cases and microwire rupture in 1 . The first detachment failure occurred in a patient with a large, wide-necked intracavernous ICA aneurysm. The primary SILK deployment was impossible given a large defect of the artery wall, though the device slipped into the aneurysm sac. After many attempts and an evaluation of collateral flow with an occlusion test, a LEO stent $(3.5 \times 35$, Balt Extrusion) was first inserted and then another FDD was placed into the first stent 1 month later (Fig. 1). The second detachment failure occurred in a patient harboring 2 mirror PCoA aneurysms. One was successfully treated; however, in the contralateral aneurysm, deployment of the FDD (FRED) failed because of a proximal calcified stenosis. Balloon-assisted coiling was then realized (Fig. 2). One patient had rupture of the Pipeline microwire, which was immediately captured with a GooseNeck device (Covidien) without any clinical problems.

Postprocedural complications occurred in 6 patients (10\%); none led to permanent clinical or neurological sequelae. Hence, we observed transient dysarthria in 2 patients, a single case of femoral pseudoaneurysm, an episode of hemoptysis after the extubation with consequent aspiration pneumonia, and vasospasm with acute intrastent aggregation in 2 patients. In the early postprocedural period, the transient dysarthria cases were treated with Abciximab (ReoPro, intravenous bolus dose of $0.25 \mathrm{mg}$ / $\mathrm{kg}$ followed by a continuous infusion of $0.125 \mu \mathrm{g} / \mathrm{kg} / \mathrm{min}$ for 12 hours), with complete recovery from the symptoms.

The carotid vasospasm required dedicated treatment: in 

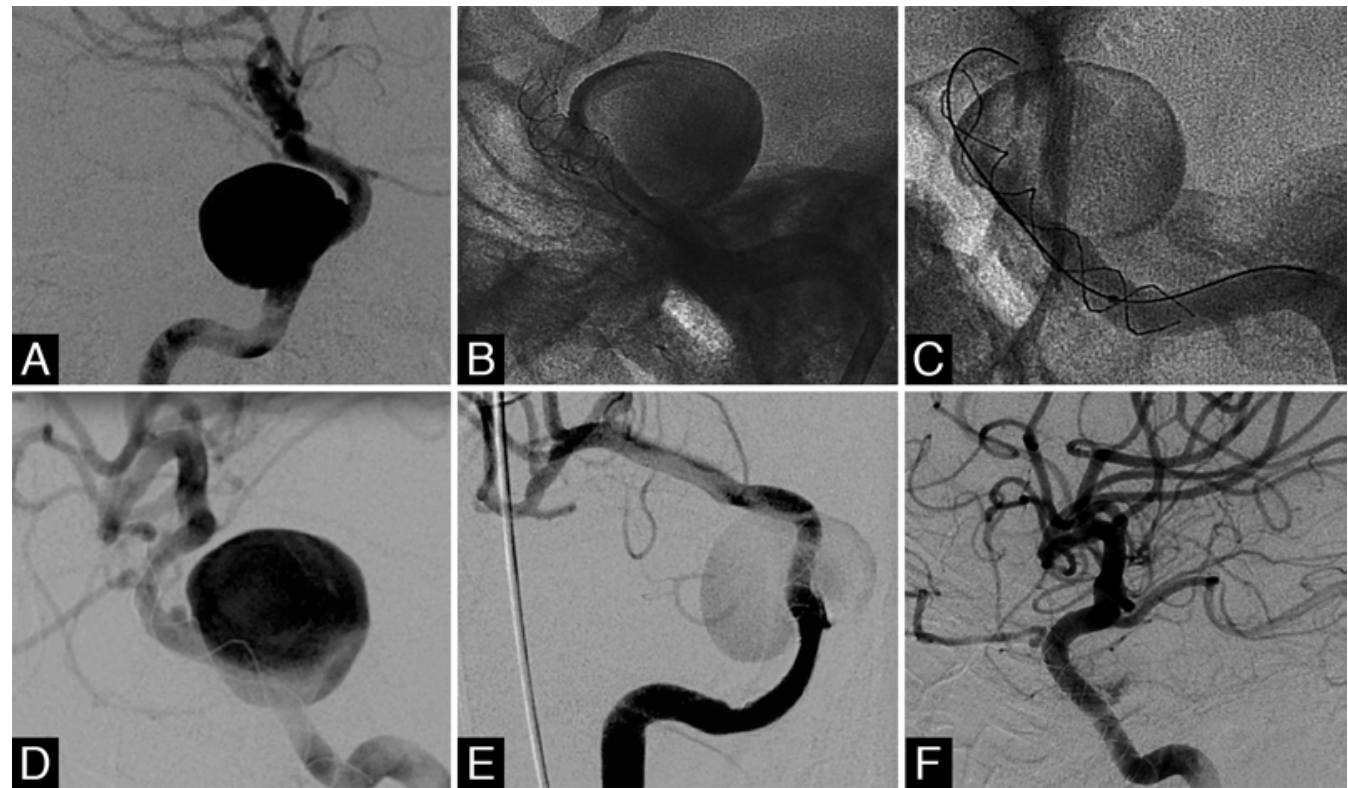

FIG. 1. Digital subtraction angiograms were obtained in a 45-year-old woman, showing a large wide-necked intracavernous ICA aneurysm (A). The primary SILK deployment was impossible given a large defect of the artery wall, although the device fell into the aneurysm sac (B); therefore, a LEO stent $(3.5 \times 35$, Balt Extrusion, $\mathbf{C})$ was first inserted, then another FDD was later placed into the first stent (D). Postprocedure DSA image (E) showing contrast stasis in the aneurysm sac. Six-month follow-up DSA image (F) showing complete occlusion of the aneurysm.

1 patient treated with the SILK device for a small carotid ophthalmic aneurysm, vasospasm occurred a few minutes after a successful procedure, with a reduction in the distal FDD's diameter. After many balloon dilation attempts, upon evaluation of an adequate contralateral flow supply in a balloon-occlusion test, the ICA was closed up with coils. From a clinical standpoint, the patient did not experience any complications related to this rescue procedure. The second case occurred in a patient with a large PCoA aneurysm that had been treated with FDD and coils; the vasospasm did not resolve after intraarterial nimodipine $(2 \mathrm{mg})$ administration and an angioplasty attempt. Again, the collateral feeders' supply was adequate so that a slight controlled hypertension regimen avoided ischemic complications. Restored flow and patency of the device with partial aneurysm occlusion was observed at 3 months, and complete aneurysm occlusion was finally observed at 12 months.

The periprocedural complications were not correlated with the type of FDD used. No delayed aneurysm rupture, no SAH or intraparenchymal hemorrhage, and no deaths occurred. At the 12- to 18-month MR follow-up, 3 patients (5\%) experienced asymptomatic cerebral infarction. It occurred at the basal ganglia in 2 cases (lenticular and head of the caudate nucleus, respectively) and in the posterior temporal lobe in 1 case (Figs. 3-5). No additional complications occurred at the longer-term follow-up ( $>24$ months).

\section{Discussion}

The aim of treatment with an FDD is to disrupt intraaneurysmal flow and favor intraaneurysmal thrombosis while preserving the patency of adjacent vessels. Flow di- version may provide a valid alternative to conventional endovascular techniques, especially when dealing with difficult-to-treat aneurysms such as wide-necked, fusiform, or giant aneurysms or those with complex morphology, cases in which flow diversion can grant higher occlusion rates, along with the possibility of preserving the parent artery. $1,2,4-10,13,17-19,22-25$ Since their introduction into clinical practice, the SILK flow diverter and PED have had excellent results, according to multiple studies. ${ }^{1,2,4-8,10,11,13}$ In addition, recent cost-effectiveness analyses have favored flow diversion over alternative endovascular treatments, particularly for large or giant aneurysms.${ }^{11}$ However, consensus has not been reached as regards the long-term stability of treatment and complication rates.

The results of our series confirm that the FDD technique is affordable and viable, depending on the aneurysm location, size, morphology, previous treatments, and type
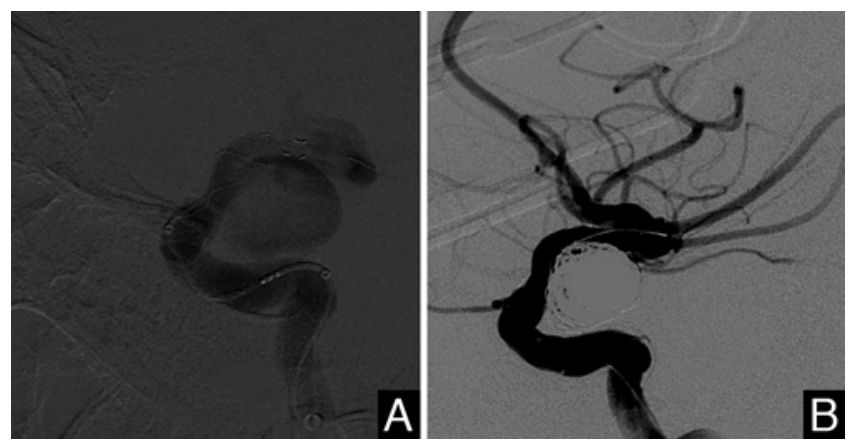

FIG. 2. A 67-year-old man had a large wide-necked ICA aneurysm at the origin of the PCoA (A). Deployment of the FRED was impossible because of calcified intracranial stenosis of the ICA siphon; therefore, aneurysm embolization with balloon-assisted coiling was performed (B). 

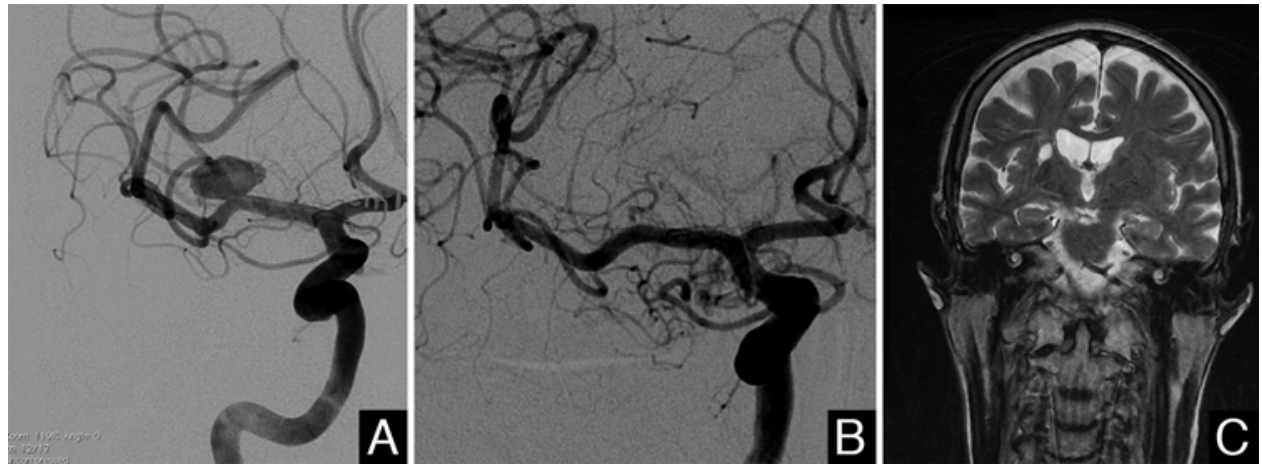

FIG. 3. Digital subtraction angiography study obtained in a 68-year-old woman harboring a right $M_{1}-M_{2}$ bifurcation aneurysm (A). The 6-month follow-up angiography (B) showed complete occlusion of the sac. The side branch covered by the PED is reduced in filling. At eighteen months postoperatively, coronal turbo spin echo T2-weighted MR image (C) showed infarction in the right lenticular nucleus.

of device used. Indeed, complete occlusion was achieved in almost all aneurysms of the ICA siphon (56 [98\%] of 57) and in all cases (100\%) located at the paraophthalmic and cavernous segments. In particular, results for aneurysms located at the cavernous segment are encouraging, showing a high occlusion rate at the latest follow-up (7 [100\%] of 7), with no morbidity. Aneurysms in this location often present with cranial nerve palsies and are difficult to treat with other endovascular techniques. As compared with other series accounting only for cavernous aneurysms treated with PED, ${ }^{21}$ our current study shows a higher occlusion rate along with no mortality and/or permanent morbidity.
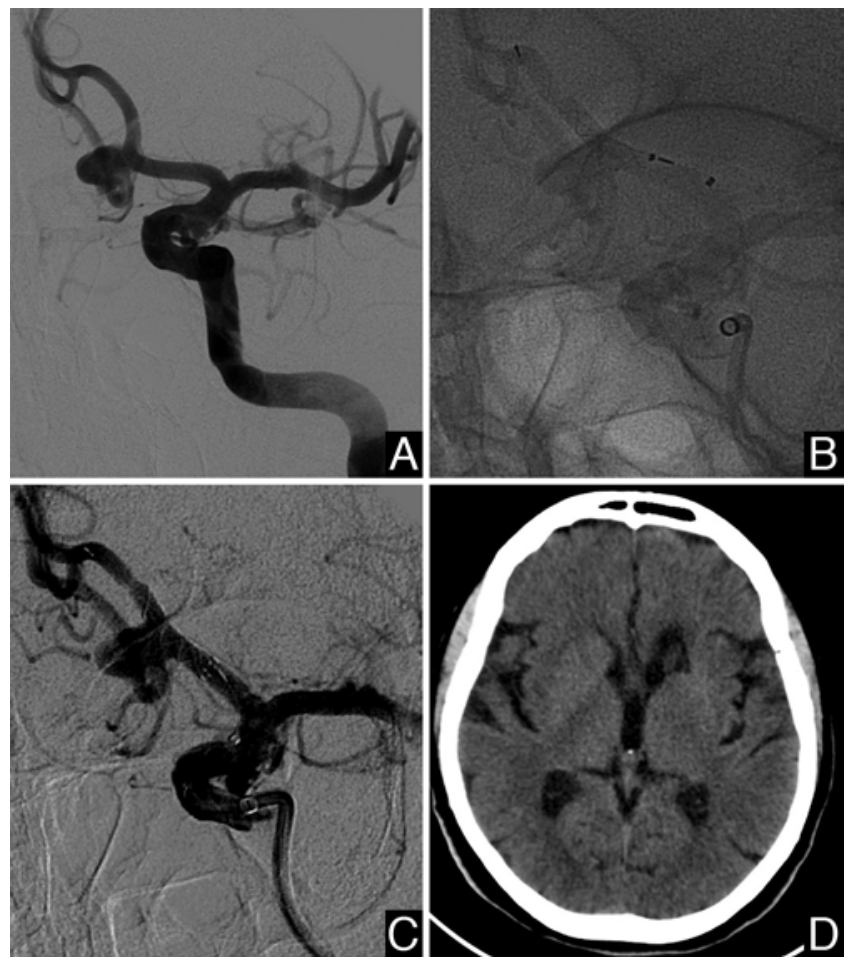

FIG. 4. Digital subtraction angiogram (A) obtained in a 67-year-old woman harboring a small ACA aneurysm at the corner of the left $A_{2}$ Nonsubtracted $(B)$ and subtracted $(\mathbf{C})$ angiographic images obtained after p64 deployment. Axial CT scan (D) obtained at 12 months, showing infarction of the left caudate head.
Above all, complete resolution or significant improvement of symptoms occurred in all symptomatic patients.

On the other hand, when dealing with paraophthalmic aneurysms, the major concerns are preserved patency of the ophthalmic artery and the avoidance of eventual ophthalmological complications. As demonstrated in a study by Durst et al. ${ }^{12}$ and confirmed by data in our study, these risks are negligible; indeed, all ophthalmic arteries covered by an FDD remained patent.

Lanzino et al. ${ }^{16}$ reported a significantly higher rate of complete occlusion for paraclinoid aneurysms in patients treated with flow diverters (76\%) than in those treated with coils (21\%), with the 2 groups presenting a similar rate of morbidity. These authors suggested that long-term followup was important to rule out flow diversion as a superior therapeutic strategy for proximal ICA aneurysms.

Moreover, as reported in large series, , ${ }^{1,2,4-10,13,17-19,22-25}$ the chance of achieving complete aneurysm occlusion with an FDD, especially in side-wall aneurysms such as the paraclinoid ones, is very high and most likely to be higher than those with coil embolization with or without balloon or stent assistance. In the present study, we observed effective, complete, and stable occlusion at the long-term follow-up, so we can attest to a certain superiority of FDD treatment for aneurysms of this location.

On the other hand, it is also noteworthy that in all 4 cases in which aneurysms showed partial occlusion at the last follow-up (1 each at the MCA, ACA, PCoA, and SCA), a side branch arising from the aneurysm sac was identified. This could have led to flow reversal, eventually increased for a "sump effect" and thus resulting in lower flow-diversion efficacy. However, all aneurysms with partial occlusion and consequent remodeling of the sac were stable at follow-up without signs of regrowth.

As regards occlusion of the parent vessel, we reported a non-negligible rate (2 [3\%] of 69 aneurysms). In 1 case, a large basilar artery aneurysm was treated with an FDD and coiling because the dual antiplatelet therapy was interrupted; in the other case, an ICA paraclinoid aneurysm treated with an FDD was complicated by a severe vasospasm, with a reduction in the distal FDD's diameter requiring rescue vessel occlusion treatment with coils. The patients in these cases did not experience any complications, and adequate support therapy avoided delayed ischemic complications. 

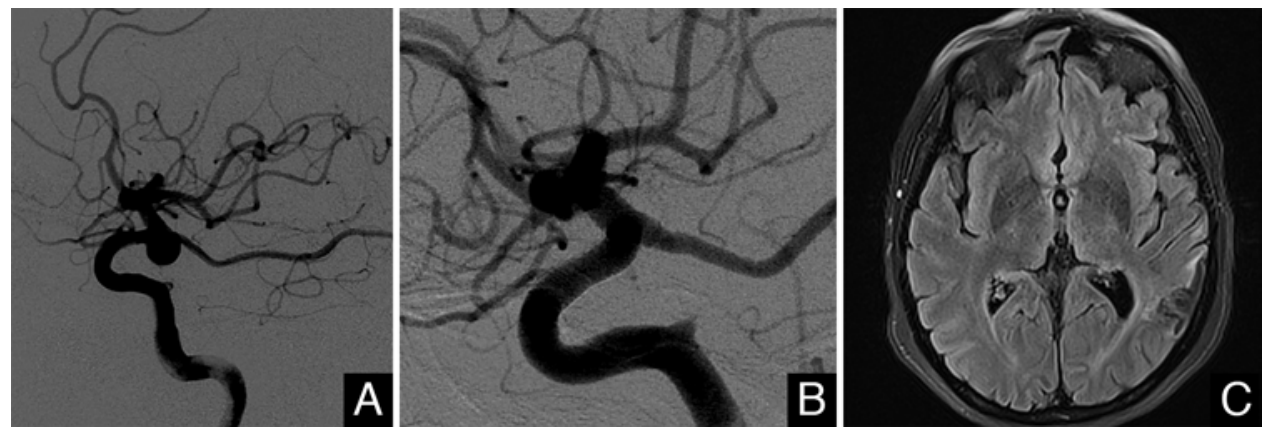

FIG. 5. Digital subtraction angiogram (A) obtained in a 58-year-old woman harboring a small PCoA aneurysm and ipsilateral fetal origin of the posterior cerebral artery. Six-month follow-up angiogram (B) obtained after PED placement, showing complete occlusion of the aneurysm sac. Twelve-month postoperative T2-weighted FLAIR image (C) showing infarction in the left posterior temporal lobe.

It should also be remembered that for aneurysms at the ICA siphon, a strict long-lasting dual antiplatelet therapy regimen is mandatory and, above all, that the ICA occlusion test should be run before the FDD treatment procedure.

As regards complications, it has been reported that ischemic stroke following FDD treatment is not common, with an incidence ranging from $4.1 \%{ }^{4}$ to $4.5 \% .^{9}$ In our series, complications at the 12- to 18-month follow-up occurred in 3 patients (5\%). One ischemic complication occurred in a patient harboring a PCoA aneurysm and an ipsilateral fetal origin of the posterior cerebral artery and can probably be attributed to a thromboembolic phenomenon. In the other 2 cases, the patients presented with aneurysms located beyond the circle of Willis (ACA and MCA, respectively) and related to perforator occlusion. This phenomenon can be logically explained by the progressive flow reduction through the covered perforators into a brain area without adequate collateral feeders. This seems to be in line with the report by Gawlitza et al., ${ }^{14}$ which noted that caliber reduction and asymptomatic occlusion of covered cortical branches and lacunar infarcts after FDD placement are common events, being reported in $41.2 \%$ of the procedures. No other complication occurred at the longerterm follow-up (> 24 months).

Owing to these data, we think it is worth discussing the results and occlusion rates of the present series in terms of the type of FDD used. In the subgroup of patients treated with the SILK flow diverter-4 patients each harboring an intracranial aneurysm-complete occlusion (OKM D) was achieved in $3(75 \%)$ of the 4 aneurysms. Intraprocedural and postprocedural technical problems occurred in 2 (50\%) of these patients: in the first case the device slipped into the aneurysm, in the second case the implanted device caused a severe vasospasm of the parent vessel, which required complete occlusion with coils. Both of these issues could have been related to a technical aspect of SILK, namely its lower radial strength. Although we abandoned this FDD in favor of other devices with superior radial force, these results could have also been affected by the surgeon's lack of experience. Indeed, the SILK diverter was adopted for treatment of the first 4 patients of this study cohort, as it was the first device to be introduced into the clinical practice.

In the subgroup of aneurysms treated with FRED (20 patients harboring 24 intracranial aneurysms), complete occlusion (OKM D) was achieved in 23 aneurysms (96\%) and partial occlusion (OKM C) was achieved in $1(4 \%){ }^{6}$ This last case was a PCoA aneurysm with an ipsilateral fetal origin of the posterior cerebral artery. Again, the presence of a side branch arising from the aneurysm sac could explain the incomplete occlusion at the latest follow-up (2 years). As regards time, complete occlusion was achieved at 3 months in 10 cases (42\%), at 6 months in $11(46 \%)$, and at 12 months in 2 (8\%); all FRED implants were patent at the last follow-up.

In the subgroup of aneurysms treated with p64 (5 patients with 6 intracranial aneurysms), complete occlusion (OKM D) was obtained in 3 aneurysms (50\%); 1 patient experienced asymptomatic PAO because of antiplatelet therapy withdrawal, and the other 2 (harboring SCA and $\mathrm{A}_{1} / \mathrm{A}_{2}$ aneurysms) showed partial occlusion (OKM C).

Analyzing the subgroup of aneurysms treated with PED (31 patients harboring 35 intracranial aneurysms), complete occlusion (OKM D) was obtained in 34 aneurysms (97\%) and partial occlusion (OKM C) in $1(3 \%)$. This last case was an MCA aneurysm located at the $\mathbf{M}_{1}-\mathbf{M}_{2}$ bifurcation, with a side branch arising from the aneurysm sac, which could have played a pivotal role in the incomplete occlusion at the last follow-up (3 years). Again, all PED implants were patent at the last follow-up.

Given these data, we determined that better occlusion rates are obtained with the PED and FRED, although in the present series the number of cases treated with SILK and p64 are limited. Furthermore, morbidity and mortality rates are similar to those reported in other series in the literature. ${ }^{5,17,18,23}$

Finally, it is worth noting that the retrospective study design, small number of included cases, and prevalence of aneurysms of the ICA siphon (83\%) and small aneurysms (78\%) could represent certain limitations.

\section{Conclusions}

Endovascular treatment with an FDD is a safe and effective option for intracranial aneurysms, allowing for higher occlusion rates as well as a low risk of complications. Hence, the patient selection process is very important to avoid treatment failure and reduce the risk of morbidity and mortality. 
In the present study, we observed effective and stable aneurysm occlusion at the long-term follow-up in patients treated with an FDD. Data in this study also suggest that ischemic complications can occur at a later stage, particularly at $12-18$ months, and can be mainly related to perforator occlusion or thromboembolic complication. On the other hand, no other ischemic or hemorrhagic complications occurred beyond 24 months.

\section{References}

1. Arrese I, Sarabia R, Pintado R, Delgado-Rodriguez M: Flow-diverter devices for intracranial aneurysms: systematic review and meta-analysis. Neurosurgery 73:193-200, 2013

2. Berge J, Biondi A, Machi P, Brunel H, Pierot L, Gabrillargues J, et al: Flow-diverter silk stent for the treatment of intracranial aneurysms: 1-year follow-up in a multicenter study. AJNR Am J Neuroradiol 33:1150-1155, 2012

3. Briganti F, Delehaye L, Leone G, Sicignano C, Buono G, Marseglia M, et al: Flow diverter device for the treatment of small middle cerebral artery aneurysms. J Neurointerv Surg 8:287-294, 2016

4. Briganti F, Leone G, Marseglia M, Mariniello G, Caranci F, Brunetti A, et al: Endovascular treatment of cerebral aneurysms using flow-diverter devices: A systematic review. Neuroradiol J 28:365-375, 2015

5. Briganti F, Leone G, Ugga L, Marseglia M, Macera A, Manto A, et al: Mid-term and long-term follow-up of intracranial aneurysms treated by the p64 Flow Modulation Device: a multicenter experience. J Neurointerv Surg 9:70-76, 2017

6. Briganti F, Leone G, Ugga L, Marseglia M, Solari D, Caranci F, et al: Safety and efficacy of flow re-direction endoluminal device (FRED) in the treatment of cerebral aneurysms: a single center experience. Acta Neurochir (Wien) 158:17451755, 2016

7. Briganti F, Napoli M, Leone G, Marseglia M, Mariniello G, Caranci $\mathrm{F}$, et al: Treatment of intracranial aneurysms by flow diverter devices: long-term results from a single center. Eur J Radiol 83:1683-1690, 2014

8. Briganti F, Napoli M, Tortora F, Solari D, Bergui M, Boccardi E, et al: Italian multicenter experience with flow-diverter devices for intracranial unruptured aneurysm treatment with periprocedural complications-a retrospective data analysis. Neuroradiology 54:1145-1152, 2012

9. Brinjikji W, Lanzino G, Cloft HJ, Siddiqui AH, Boccardi E, Cekirge S, et al: Risk factors for ischemic complications following pipeline embolization device treatment of intracranial aneurysms: results from the IntrePED Study. AJNR Am J Neuroradiol 37:1673-1678, 2016

10. Brinjikji W, Murad MH, Lanzino G, Cloft HJ, Kallmes DF: Endovascular treatment of intracranial aneurysms with flow diverters: a meta-analysis. Stroke 44:442-447, 2013

11. Colby GP, Lin LM, Paul AR, Huang J, Tamargo RJ, Coon $\mathrm{AL}$ : Cost comparison of endovascular treatment of anterior circulation aneurysms with the pipeline embolization device and stent-assisted coiling. Neurosurgery 71:944-950, 2012

12. Durst CR, Starke RM, Clopton D, Hixson HR, Schmitt PJ, Gingras JM, et al: Endovascular treatment of ophthalmic artery aneurysms: ophthalmic artery patency following flow diversion versus coil embolization. J Neurointerv Surg 8:919-922, 2016

13. Fischer S, Vajda Z, Aguilar Perez M, Schmid E, Hopf N, Bäzner H, et al: Pipeline embolization device (PED) for neurovascular reconstruction: initial experience in the treatment of 101 intracranial aneurysms and dissections. Neuroradiology 54:369-382, 2012

14. Gawlitza M, Januel AC, Tall P, Bonneville F, Cognard C:
Flow diversion treatment of complex bifurcation aneurysms beyond the circle of Willis: a single-center series with special emphasis on covered cortical branches and perforating arteries. J Neurointerv Surg 8:481-487, 2016

15. Kallmes DF, Ding YH, Dai D, Kadirvel R, Lewis DA, Cloft HJ: A new endoluminal, flow-disrupting device for treatment of saccular aneurysms. Stroke 38:2346-2352, 2007

16. Lanzino G, Crobeddu E, Cloft HJ, Hanel R, Kallmes DF: Efficacy and safety of flow diversion for paraclinoid aneurysms: a matched-pair analysis compared with standard endovascular approaches. AJNR Am J Neuroradiol 33:2158-2161, 2012

17. Lubicz B, Van der Elst O, Collignon L, Mine B, Alghamdi F: Silk flow-diverter stent for the treatment of intracranial aneurysms: a series of 58 patients with emphasis on long-term results. AJNR Am J Neuroradiol 36:542-546, 2015

18. Maimon S, Gonen L, Nossek E, Strauss I, Levite R, Ram Z: Treatment of intra-cranial aneurysms with the SILK flow diverter: 2 years' experience with 28 patients at a single center. Acta Neurochir (Wien) 154:979-987, 2012

19. Martínez-Galdámez M, Pérez S, Vega A, Ruiz P, Caniego JL, Bárcena E, et al: Endovascular treatment of intracranial aneurysms using the Pipeline Flex embolization device: a case series of 30 consecutive patients. J Neurointerv Surg 8:396-401, 2016

20. O'Kelly CJ, Krings T, Fiorella D, Marotta TR: A novel grading scale for the angiographic assessment of intracranial aneurysms treated using flow diverting stents. Interv Neuroradiol 16:133-137, 2010

21. Puffer RC, Piano M, Lanzino G, Valvassori L, Kallmes DF, Quilici L, et al: Treatment of cavernous sinus aneurysms with flow diversion: results in 44 patients. AJNR Am J Neuroradiol 35:948-951, 2014

22. Saatci I, Yavuz K, Ozer C, Geyik S, Cekirge HS: Treatment of intracranial aneurysms using the pipeline flow-diverter embolization device: a single-center experience with longterm follow-up results. AJNR Am J Neuroradiol 33:14361446, 2012

23. Shankar JJ, Tampieri D, Iancu D, Cortes M, Agid R, Krings T, et al: SILK flow diverter for complex intracranial aneurysms: a Canadian registry. J Neurointerv Surg 8:273-278, 2016

24. Wagner A, Cortsen M, Hauerberg J, Romner B, Wagner MP: Treatment of intracranial aneurysms. Reconstruction of the parent artery with flow-diverting (Silk) stent. Neuroradiology 54:709-718, 2012

25. Yu SC, Kwok CK, Cheng PW, Chan KY, Lau SS, Lui WM, et al: Intracranial aneurysms: midterm outcome of pipeline embolization device - a prospective study in 143 patients with 178 aneurysms. Radiology 265:893-901, 2012

\section{Disclosures}

Dr. Briganti serves as proctor for Covidien.

\section{Author Contributions}

Conception and design: Briganti. Acquisition of data: Briganti, Leone. Analysis and interpretation of data: Briganti, de Divitiis, Solari. Drafting the article: Leone. Critically revising the article: Briganti, Leone, Solari, Cappabianca. Reviewed submitted version of manuscript: Cappabianca. Administrative/technical/material support: de Divitiis. Study supervision: de Divitiis, Cappabianca.

\section{Correspondence}

Francesco Briganti, Department of Advanced Biomedical Sciences, Unit of Interventional Neuroradiology, Federico II University, Via S. Pansini, 5, Naples 80131, Italy. email: frabriga@unina.it. 